\title{
ARTICULOS OR
}

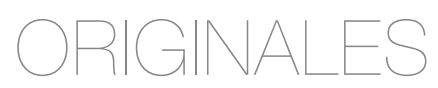

\section{Una aproximación a las políticas laborales sobre identidad de género en multinacionales en México}

\author{
JOSÉ CARLOS VÁZQUEZ PARRA* \\ JUAN ALBERTO AMÉZQUITA ZAMORA** \\ NATALIA ROCHA DÍAZ***
}

* Doctor en Estudios Humanísticos. Instituto Tecnológico de Estudios Superiores de Monterrey, Campus Guadalajara, Zapopan, México. E-mail: jcvazquezp@tec.mx. ORCID: 00oo-0001-9197-7826. Google Scholar: https://scholar.google.com.ua/citations?hl=en\&user=RhQ PwMAAAAJ. Scopus Author ID: https://www.scopus.com/authid/detail.uri?authorld=55871913100.

** Doctor en Estudios Internacionales. Instituto Tecnológico de Estudios Superiores de Monterrey, Campus Guadalajara, Zapopan, México. E-mail: ja.amezquita@tec.mx. ORCID: oooo-0oo2-0297-6416. Google Scholar: https://scholar.google.com/ citations?user=1vQSU94AAAAJ\&hl=es.

*** Maestra en Estudios Humanísticos. Instituto Tecnológico de Estudios Superiores de Monterrey, Campus Guadalajara, Zapopan, México. E-mail: natalia.rocha@tec.mx. ORCID: oooo-0002-6374-108X. Google Scholar: https://scholar.google.com/ citations? user=P6N4KHYAAAAJ\&hl=en \&authuser $=1$. 
COMO CITAR ESTE ARTÍCULO

How to cite this article:

Vázquez, J.C., Amézquita, J.A. y Rocha, N. (2021). Una aproximación a las políticas laborales sobre identidad de género en multinacionales en México. Revista Perspectiva Empresarial, 8(1), 86-100.

Recibido: 08 de enero de 2021 Aceptado: 30 de junio de 2021
RESUMEN Objetivo. Determinar si un grupo muestra de empresas multinacionales situadas en México implementan políticas laborales de inclusión apegadas a lo que están obligadas por ley en sus Naciones de origen. Metodología. Se hizo un análisis de contenidos de los reportes de responsabilidad social de las empresas, así como de los derechos laborales internos hacia el colectivo LGBTI. Resultados. El análisis arrojó poca concordancia entre las legislaciones de los países de las empresas y sus políticas internas relacionadas con la orientación sexual y la identidad de género de sus colaboradores. Conclusiones. Este texto permite apreciar que las empresas seleccionadas, al no ser congruentes entre sus normativas locales e internacionales, pueden gestar incertidumbre en sus trabajadores con respecto al derecho a la no discriminación por motivos de su identidad de género.

PALABRAS CLAVE diversidad, discriminación, identidad de género, transgénero, derechos humanos.

\section{An approach to labor policies on gender identity in Mexican multinationals}

ABSTRACT Objective. To determine whether a sample group of multinational companies located in Mexico implement labor market inclusion policies in accordance with what they are required by law in their countries of origin. Methodology. A content analysis of the companies' social responsibility reports was carried out, as well as an analysis of the internal labor rights of the LGBTI community. Results. The analysis showed little consistency between the legislation of the companies' countries and their internal policies related to the sexual orientation and gender identity of their employees. Conclusions. This work shows that the selected companies, by not being congruent with their local and international regulations, can generate uncertainty in their workers with respect to the right to non-discrimination on the grounds of their gender identity.

KEYWORDS Diversity, discrimination, gender identity, transgender, human rights. 


\section{Uma abordagem às políticas trabalhistas sobre identidade de gênero em multinacionais no México}

RESUMO Objetivo. Determine se um grupo de amostra de empresas multinacionais localizadas no México implementa políticas de inclusão trabalhista que atendam ao que é exigido por lei em seus países de origem. Metodologia. Foi feita uma análise do conteúdo dos relatórios de responsabilidade social corporativa, bem como dos direitos trabalhistas internos da comunidade LGBTI. Resultados. A análise encontrou pouca concordância entre as legislações dos países das empresas e suas políticas internas relacionadas à orientação sexual e identidade de gênero de seus funcionários. Conclusões. Este texto permite avaliar que as empresas selecionadas, por não serem congruentes entre suas regulamentações locais e internacionais, podem gerar incertezas em seus trabalhadores quanto ao direito à não discriminação em razão de sua identidade de gênero.

PALAVRAS CHAVE diversidade, discriminação, identidade de gênero, transgênero, direitos humanos. 


\section{Introducción}

Uno de los mayores retos que enfrentan las empresas contemporáneas tiene que ver con el manejo de su capital humano. A nivel internacional, la atención a sus grupos de interés internos ha motivado que las organizaciones planteen múltiples programas y proyectos de responsabilidad social para mejorar las condiciones laborales de sus colaboradores (Szyndlar and Wasikiewicz-Firlej, 2019). Un tema central en esas iniciativas tiene que ver con las políticas laborales, las cuales deben ser incluyentes y respetuosas de las características y preferencias individuales de cada uno de estos grupos de interés. Las empresas deben estar listas para poder brindar a sus colaboradores entornos en los que no solo puedan trabajar y desarrollarse profesionalmente, sino también ámbitos en los que consigan alcanzar un equilibrio entre su vida personal y laboral (ADIL, 2018).

Lamentablemente la rentabilidad, la búsqueda de mayores utilidades y la lucha por la eficiencia pueden llevar a que las empresas se enfrenten a situaciones problemáticas que pongan en tela de juicio las políticas que dicen estar implementando para garantizar la salud, la seguridad y la calidad de vida de sus empleados y empleadas, lo cual puede ir desde la falta de control sobre posibles condiciones laborales extremas o peligrosas hasta el desconocimiento de los derechos fundamentales de los que en ellas laboran (Lamond, 2018).

El presente artículo analiza la implementación de políticas laborales apegadas a normas nacionales e internacionales relacionadas con el derecho a la no discriminación por motivo de identidad de género, por tratarse de una categoría todavía muy descuidada en las regulaciones y políticas empresariales (WORLD Policy Analysis Center, 2020). Para lograrlo se hace una aproximación a la normativa contra la discriminación desde una visión de derechos humanos. De manera concreta, y después de plantear un marco de análisis, se reflexiona sobre las políticas laborales de empresas multinacionales situadas en México - firmantes del Pacto Mundial-que son originarias de Naciones que cuentan con leyes en contra dela discriminación laboral por motivos de identidad de género. Como conclusión se espera identificar si este tipo de normas efectivamente tienen un impacto en las políticas internas de estas organizaciones.

\section{Marco teórico: de los derechos humanos a las normas laborales}

\section{Definiciones fundamentales: orientación sexual, identidad de género, expresión de género y características sexuales ${ }^{1}$}

El punto de partida obligatorio para este estudio es dejar en claro la diferencia entre sexo y género. Se llama 'sexo' a "las diferencias biológicas entre el hombre y la mujer" (CEDAW, 2010, p. 2); es decir que cuando se refiere al sexo se habla de "una construcción biológica que se refiere a las características genéticas, hormonales, anatómicas y fisiológicas sobre cuya base una persona es clasificada como macho o hembra al nacer" (CIDH, 2012,p. 3). Esta definición podría ser considerada como una declaración de que dado los cuerpos y atendiendo al sexo tan solo son posibles dos opciones para clasificar a las personas: ser varón o ser mujer. Sin embargo no se puede dejar de lado el hecho de que "hay personas cuyos cuerpos presentan factores que hacen que su configuración genética, gonádica, morfológica u hormonal difiera de lo que culturalmente suele entenderse estrictamente como el sexo 'masculino' o el 'sexo femenino'” (Suprema Corte de Justicia de la Nación, 2014 , p. 13). Esas personas que no cumplen los estándares normativos vigentes para distinguir los cuerpos sexuados de los hombres y las mujeres son llamadas intersexuales.

Precisamente para romper con esta asociación naturalizada del sexo con la mencionada clasificación binaria, en documentos recientes de las Naciones Unidas (como, por ejemplo, los Principios de Yogyakarta Plus 10 y el reporte Born Free and Equal de la OHCHR) se prefiere la terminología de características sexuales para hablar de lo que aquí

\footnotetext{
${ }^{1}$ Los autores agradecen al Dr. Armando Díaz Camarena por la orientación para enfocar y escribir esta sección del artículo.
} 
se ha llamado sexo; entendiendo por ellas a "las características físicas de cada persona relacionadas con el sexo, incluyendo los genitales y otros elementos de la anatomía sexual y reproductiva, los cromosomas, las hormonas y los caracteres sexuales secundarios que se manifiestan en la pubertad" (OHCHR, 2019, p. 5); lo anterior, coincide con lo que se había dicho antes sobre el sexo. Utilizando la terminología de características sexuales se evita que el uso de la palabra 'sexo' excluya tácitamente a las personas intersexuales, quienes se estima que constituyen el $2 \%$ de la población mundial (Díaz Camarena, 2017).

Conviene ahora definir el género, el cual "se refiere a las identidades, las funciones y los atributos construidos socialmente de la mujer y el hombre y al significado social y cultural que la sociedad atribuye a esas diferencias biológicas" (CEDAW, 2010, p. 2). Esta noción incluye cómo son entendidos y entendidas los hombres y las mujeres, las funciones que se les atribuyen socialmente (por ejemplo, los roles de proveedores y cuidadoras), las características que se les asignan (como ser racional o emocional) y otras diferencias culturales que se les atribuyen (como los nombres que se consideran como propios de los hombres y propios de las mujeres); sin olvidar que por razones de género también se justifican las formas de dependencia, dominio, superioridad o inferioridad entre hombres y mujeres que en muchas sociedades son determinadas por las diferencias biológicas, dejando de lado que se trata de constructos culturales (Flores, 2016).

La distinción entre sexo - o características sexuales-y género es de gran trascendencia para comprender que "no hay una correlación necesaria entre el cuerpo con el que una persona nacey la personalidad que desarrolla o las funciones sociales que cumple" (Suprema Corte de Justicia de la Nación, 2014, p. 13). Así pues, en el caso del sexo o las características sexuales se puede afirmar que la biología no es destino ni genera por sí misma obligaciones de ningún tipo que determinen la existencia de los seres humanos.

En segundo término es importante abordar las nociones de identidad de género y la expresión de género. Según los Principios de Yogyakarta de 2007, la identidad de género designa la vivencia interna e individual del género como cada persona la siente profundamente; esta podría corresponder o no con el sexo asignado al momento del nacimiento, incluyendo la vivencia personal del cuerpo (que podría involucrar la modificación de la apariencia o la función corporal a través de medios médicos, quirúrgicos o de otraíndole, siempre que la misma sea libremente escogida) y otras expresiones de género tales como la vestimenta, el modo de hablar y los modales.

La identidad de género se refiere a la forma en que cada persona se asume a sí misma en relación con la asignación de su sexo al nacer, lo cual puede coincidir o no con esa asignación. Se utilizan los términos trans o transgénero para referirnos precisamente a todas aquellas personas que no se identifican a sí mismas con el sexo que socialmente se les asignó al nacer o que se consideran fuera de la clasificación binaria de masculino/femenino o son percibidas como personas atípicas en el terreno del género por su apariencia o sus rasgos (Vijlbrief, Saharso and Ghorashi, 2020; Matthews, 2020). En esta categoría se encuentran los hombres trans y las mujeres trans (personas que se identifican con un género distinto al sexo que se les asignó al nacer), las personas transexuales (quienes optan por una intervención médica ya sea hormonal y/o quirúrgica para adecuar su condición físicabiológica a su orientación de género), el travestismo, las personas queer, las personas no binarias entre otras. Por otra parte, hoy en día, es común el uso del adjetivo cisgénero para referirse a las personas que tienen una identidad de género que coincide con el sexo que se les asignó al nacer (OHCHR, 2019).

La expresión de género es una dimensión de la identidad de género que designa la manifestación externa de aquellos rasgos culturales que nos permiten identificar el género de una persona a través de sus patrones de comportamiento, su apariencia física -incluyendo el vestido, el peinado, los accesorios o los cosméticos-, la forma de hablar y sus gestos habituales particulares, lo mismo que los nombres y pronombres con que se refiere a sí misma. De este modo la expresión de género se referiría a todos aquellos elementos de la presencia y actuación de las personas, de su manifestación frente a las otras personas en cualquier interacción social, que permite a los demás miembros de la sociedad identificarlas desde la perspectiva del género al margen de cómo se identifica cada uno y cada una; mientras que 
la identidad de género sería la forma en que cada persona se asume a sí misma, independientemente de la interpretación de los demás (Suprema Corte de Justicia de la Nación, 2014).

Finalmente se debe de hablar de orientación sexual, la cual se refiere a:

capacidad de cada persona de sentir una profunda atracción emocional, afectiva y sexual por personas de un género diferente al suyo, o de su mismo género, o de más de un género, así como a la capacidad de mantener relaciones íntimas y sexuales con estas personas. (Principios de Yogyakarta, 2007, p. 6, nota 1$)$

Así pues, se tienen tres posibilidades en la orientación sexual: cuando las personas se sienten atraídas y pueden establecer relaciones con personas de su mismo género se habla de personas homosexuales, las cuales serán lesbianas si se trata de mujeres y gay si se trata de hombres (Wilson and Gianella-Malca, 2019). Si las personas se sienten atraídas y pueden establecer relaciones con personas de un género distinto al suyo se habla de personas heterosexuales; mientras que se habla de bisexuales cuando se hace referencia a las personas que experimentan la atracción y pueden establecer relaciones con personas de su mismo género y de un género distinto al suyo. Las siglas que se usan para aglutinar a todas aquellas personas cuya identidad de género, preferencia sexual o características sexuales se alejan de la distinción binaria varón-mujer o de la orientación sexual de la heterosexualidad son precisamente LGBTI (lesbianas, gays, bisexuales, trans, intersexuales) (Jiménez Bolaños, 2014).

A partir de lo dicho anteriormente se puede señalar que las categorías que se han definido son independientes entre sí, de modo que es necesario superar el concepto erróneo de que podría o debería existir una correlación natural entre ellas. Así como no existe una relación necesaria entre características sexuales y género, no existe una correlación natural ni necesaria entre orientación sexual, identidad de género y expresión de género puesto que una persona trans (por ejemplo) puede tener cualquier orientación sexual o sus características sexuales no estar limitadas a un conjunto en particular. Por otra parte, las personas intersexuales pueden tener cualquier orientación sexual o identidad de género; entretanto las personas cisgénero pueden tener cualquier orientación sexual y las personas lesbianas, gay o bisexuales pueden tener cualquier identidad de género y características sexuales (OHCHR, 2019).

\section{La protección de los derechos humanos de las personas LGBTI}

Cuando se habla de los derechos humanos de la población LGBTI no se hace referencia a un grupo especial de derechos humanos sino que se está hablando de los mismos derechos humanos que se deben reconocer a todas las personas, incluidos los miembros de esta comunidad. Considerando lo anterior, se impone la siguiente pregunta: si no se pide un reconocimiento especial de derechos humanos para ese colectivo, si se está hablando de los mismos derechos humanos, entonces ¿por qué se debe hacer esa reivindicación y por qué hablar de derechos humanos para ese colectivo humano?

Para responder a esta cuestión hay que decir que el reclamo es oportuno, necesario e incluso urgente, por la sencilla razón de que las personas LGBTI siguen sufriendo en todo el mundo una cantidad inconcebible de abusos entre los que podemos mencionar:

palizas brutales, violencia sexual y asesinatos, incitación al odio, criminalización, detención y aprisionamiento arbitrario, abusos en entornos médicos, estigma generalizado, acoso, hostigamiento y discriminación en el trabajo y el hogar, así como en el acceso a la educación, la salud, la vivienda y los servicios públicos. (OHCHR, 2019, p. vii)

La protección de los derechos humanos de las personas LGBTI se fundamenta en el principio de igualdad y no discriminación, ya que es "la base del sistema internacional de protección de los derechos humanos, es un principio transversal y se encuentra consagrado en los diversos instrumentos internacionales desde la Carta delas Naciones Unidas hasta los tratados básicos de derechos humanos" (OACDH, 2013, p. 1). En efecto, el artículo 1 de la Declaración Universal de los Derechos Humanos establece que "todos los seres humanos nacen libres e iguales en dignidad y derechos". Esta declaración inequívoca exige que todos los derechos enunciados en los distintos instrumentos internacionales de 
derechos humanos sean reconocidos para todas las personas por igual; esto implica que los Estados están obligados a velar porque en su jurisdicción las leyes, políticas y programas gubernamentales eliminen cualquier forma de discriminación, así como todas las actividades de los particulares en el ámbito público y las relaciones sociales (Ball, 2019).

Por su parte el artículo 2.2 del Pacto Internacional de Derechos Económicos, Sociales y Culturales -PIDESC - requiere el compromiso por parte de los Estados de "garantizar el ejercicio de los derechos que en él se enuncian, sin discriminación alguna por motivos de raza, color, sexo, idioma, religión, opinión política o de otra índole, origen nacional o social, posición económica, nacimiento o cualquier otra condición social", lo cual es reiterado en el artículo 2.1 del Pacto Internacional de Derechos Civiles y Políticos -PIDCP-. Además, el artículo 26 del PIDCP establece que todas las personas son iguales ante la leyy tienen derecho a la misma protección jurídica; de manera que cualquier forma de discriminación se encuentra prohibida.

Aunque la identidad de género y la orientación sexual no están mencionadas explícitamente como motivos de discriminación que deben ser rechazados, diversos Órganos de Tratados de las Naciones Unidas han establecido que ambas categorías se encuentran incluidas en la mención "o cualquier otra condición social".

En relación con el fundamento de la protección de las personas LGBTI y sus derechos humanos la CIDH (2012) —además de reiterar las razones que se han señalado antes para proteger los derechos de las personas LGBTI contra toda forma de discriminación-ha añadido la consideración de que la orientación sexual, la identidad de género y la expresión de género son "componentes fundamentales de la vida privada de las personas" (p. 8), por lo que deben ser protegidas de toda invasión o agresión abusiva o arbitraria tanto por parte del Estado como por parte de terceros. A fin de cuentas, también plantea la CIDH que del derecho a la vida privada se sigue la necesidad de garantizar que las esferas de la intimidad de las personas no sean invadidas ni por el Estado ni por nadie más (Margarit, 2019). Entre esas esferas protegidas se tiene: la capacidad para desarrollar la propia personalidad y aspiraciones y determinar su propia identidad, así como campos de actividad de las personas que son propios y autónomos de cada quien, tales como sus decisiones, sus relaciones interpersonales y familiares y su hogar. (CIDH, 2012, p. 8)

Y para cualquiera de esas esferas es determinante la orientación sexual, la identidad de género, la expresión de géneroy se podría añadir a las características sexuales de cada persona.

\section{Normas nacionalese internacionales contra la discriminación laboral}

Aquí se analizan específicamente normas que se refieren al derecho al trabajo y al empleo de personas LGBTI. Respecto del derecho al trabajo, el principio No. 12 de los Principios de Yogyakarta señala que "toda persona tiene derecho al trabajo digno y productivo, a condiciones equitativas y satisfactorias de trabajo y a la protección contra el desempleo"; esto sin discriminación por motivos de orientación sexual e identidad de género, añadiendo actualmente a la expresión de género y/o características sexuales. El mismo principio establece que los Estados deben adoptar todas las medidas necesarias - legislativas, administrativas o de otra índole-para garantizar que la discriminación por los motivos señalados sea eliminada en el empleo público y privado tanto en la contratación como en la capacitación promoción, despidos, condiciones de trabajo o remuneración (Brown and Scott, 2019). También deben los Estados garantizar a las personas LGBTI la igualdad de oportunidades en todos los ámbitos del servicio público, incluyendo la policía y las fuerzas armadas. Asimismo, deberán promover los programas de capacitación y sensibilización necesarios para contrarrestar las actitudes negativas y discriminatorias (Corrales, 2017).

Por su parte el reporte Born Free and Equal, además de reiterar las ideas antes explicadas, señala también que es obligación de los Estados legislar para criminalizar y castigar el acoso y el hostigamiento sexual; igualmente que los otros mecanismos que se puedan adoptar en el lugar 
de trabajo y que hagan una referencia explícita al hostigamiento por motivos de orientación sexual, identidad de género y características sexuales. El mismo reporte señala que para 2016:

sesenta y siete Estados otorgaban protección contra la discriminación en el empleo por motivos de orientación sexual, mientras que solo 20 protegían contra la discriminación basada en la identidad/expresión de género, y solo tres protegían a la gente intersexuales de la discriminación. (OACDH, 2019, p. 65)

Como puede verse, todavía hay mucho por hacer para que la legislación de los países prohíba las prácticas discriminatorias por los motivos señalados.

Según datos del WORLD Policy Analysis Center en la última década son cada vez más numerosos los países que incluyen directrices en contra de la discriminación en sus políticas y en su normativa laboral en busca de dar mayor relevancia a la inclusión y al respeto a la diversidad que son consideradas como políticas necesarias en todas las organizaciones. Es usual encontrar en las normas laborales declaraciones que señalan que en las empresas se encuentra prohibido cualquier tipo de discriminación, sin embargo es relevante poner atención a los grupos a los que de manera expresa se hace referencia (Dario et al., 2019).

En la mayoría de estas declaraciones se puede encontrar una protección explícita contra la discriminación por motivos de género, edad, raza, religión y últimamente una ampliación a la experiencia y orientación sexual (Alarcon Andia, Castillo Montero y Gastelú Ledezma, 2018). Aunque el WORLD Policy Analysis Center ha planteado que en las políticas de protección contra la discriminación sigue siendo poco considerada la identidad de género.

Así, el mapa mundial de protección contra la discriminación en promociones o destituciones de trabajadores LGBTI publicado por el WORLD Policy Analysis Center identifica solo 16 países que dentro de sus leyes laborales incluyen señalamientos en contra de la discriminación por motivo de identidad de género (tabla 1).

Tabla 1. Países con legislaciones contra la discriminación por identidad de género

\begin{tabular}{ll}
\multicolumn{2}{l}{$\begin{array}{c}\text { Leyes contra la discriminación por orientación sexual e } \\
\text { identidad de género }\end{array}$} \\
\hline Portugal & Hungría \\
\hline Reino Unido & Eslovaquia \\
\hline Francia & Republica Checa \\
\hline Finlandia & Eslovenia \\
\hline Noruega & Bélgica \\
\hline Suecia & Luxemburgo \\
\hline Australia & Albania \\
\hline Serbia & Liberia \\
\hline
\end{tabular}

Fuente: elaboración propia por parte de los autores.

Las normas consideradas por el WORLD Policy Analysis Center incluyen a las prohibiciones o protecciones legislativas explícitas ante la discriminación relacionada con promociones, desarrollo, aspectos disciplinarios, contrataciones, destituciones y evaluación del desempeño laboral, es decir, el ciclo completo de la relación laboral de la persona con la empresa.

Es importante señalar, además, que el WORLD Policy Analysis Center pone especial atención en diferenciar las legislaciones que protegen contra la discriminación por orientación sexual de aquellas que incluyen la identidad de género como motivo rechazable de discriminación; considerando las categorías, variables o posibilidades que puede adoptar la identidad de género tales como la expresión, la reasignación o la confirmación de género, el transgenerismo y el transexualismo y la consideración de las personas hijra o kothi².

Por otro lado la normativa puede ser clasificada como concreta o general, pues en algunos casos las regulaciones de las empresas pueden incluir políticas que establecen una protección en contra

\footnotetext{
${ }^{2}$ Se comprende por la comunidad hijra o kothi a las personas consideradas de un tercer género en la India. La mayoría son hombres o intersexuales, quienes se refieren a sí mismos en femenino y suelen vestir prendas femeninas.
} 
de la discriminación en razón de la identidad de género y sus categorías asociadas; aunque también se dan casos en los que simplemente se determine una protección amplia contra todas las formas de discriminación en el trabajo.

Si bien la lista de países que incluyen normas que protegen contra la discriminación por identidad de género es bastante limitada (tabla 1), el número de empresas que proceden de esas Naciones es bastante amplio; pues en su mayoría son consideradas de ingreso alto, por lo que se calcula que en este período de tiempo tuvieron un ingreso en el PIB per cápita de \$US 12236 o más por año (Badgett y Sell, 2018). Este buen desempeño económico se ve reflejado en la amplia participación económica que suelen tener en Naciones con bajo o medio desarrollo tanto con inversión directa como indirecta.

De los 16 países que cuentan con estas normas tan solo Liberia se califica como de ingresos bajos, Albania y Serbia de ingresos medios y los demás como países de ingresos altos. Un dato adicional que es digno de ser mencionado según los datos encontrados: hay una relación directa entre el nivel de ingresos y las políticas o leyes contra la discriminación por orientación sexual e identidad de género dado que a mayor nivel de ingreso de los países, igualmente se tiene una mayor presencia de este tipo de normas laborales (tabla 2).

Tabla 2. Relación del nivel de ingreso por país y la presencia de normas contra la discriminación por orientación sexual e identidad de género

\begin{tabular}{llll}
\hline \multicolumn{1}{c}{ Nivel de ingresos } & Sin protección & \multicolumn{1}{c}{$\begin{array}{c}\text { Normas sobre orientación } \\
\text { sexual }\end{array}$} & $\begin{array}{c}\text { Normas sobre identidad de } \\
\text { género }\end{array}$ \\
\hline Países con ingresos bajos & $94 \%$ & $3 \%$ & $3 \%$ \\
\hline Países con ingresos medios & $79 \%$ & $19 \%$ & $2 \%$ \\
\hline Países con ingresos altos & $44 \%$ & $32 \%$ & $24 \%$ \\
\hline
\end{tabular}

Fuente: elaboración propia por parte de los autores.

Por otro lado es relevante considerar que existe una clara tendencia en cuanto a las regiones geográficas en donde se ubican estos países, siendo Europa la que tiene una mayor cantidad de Estados que incluyen alguno de estos dos tipos de normas como parte de sus políticas laborales nacionales; mientras que en regiones como Asia del Sur, estas políticas son aún inexistentes (tabla 3).

Tabla 3. Relación entre región geográfica y la presencia de normas contra la discriminación por orientación sexual e identidad de género

\begin{tabular}{llll}
\hline \multicolumn{1}{c}{ Región geográfica } & Sin protección & $\begin{array}{c}\text { Normas sobre } \\
\text { orientación sexual }\end{array}$ & \multicolumn{1}{c}{$\begin{array}{c}\text { Normas sobre } \\
\text { identidad de género }\end{array}$} \\
\hline América & $80 \%$ & $20 \%$ & $0 \%$ \\
\hline Asía del Este y Pacífico & $83 \%$ & $13 \%$ & $3 \%$ \\
\hline Europa y Asia Central & $30 \%$ & $42 \%$ & $28 \%$ \\
\hline Oriente Medio y Norte de África & $95 \%$ & $5 \%$ & $0 \%$ \\
\hline Asia del Sur & $100 \%$ & $0 \%$ & $0 \%$ \\
\hline África Subsahariana & $88 \%$ & $10 \%$ & $2 \%$ \\
\hline
\end{tabular}


Como se puede apreciar en las diferentes tablas, aún existe un claro camino por recorrer en la adopción de políticas laborales que protejan contra la discriminación por orientación sexual e identidad de género.

En cuanto a la protección contra la discriminación en México, todas las personas tienen derecho al trabajo digno que elijan; esto implica, según la Observación General No. 18 del Comité de Derechos Económicos, Sociales y Culturales, "el derecho a no ser privado injustamente de empleo". Este derecho está consagrado en la Constitución (artículos 5 y 123), la Ley Federal del Trabajo (artículos 2, 3, 46, 47 y 133) y la Ley Federal para Prevenir y Eliminar la Discriminación (artículos 1, 4, 5, 6, 7 y 9), por solo considerar la legislación federal. El derecho al trabajo protege a las personas a lo largo de "todo el proceso laboral: desde la oferta y contratación, pasando por el ingreso, la permanencia en el trabajo, la promoción y el despido" (Suprema Corte de Justicia de la Nación, 2014, p. 80).

En relación con las personas LGBTI este derecho exige que no sean discriminadas en ningún momento por su orientación sexual, su identidad de género, su expresión de género o sus características sexuales sino que lo que debe ser juzgado en todo lo referente a las relaciones laborales - en sus distintos momentos- no es otra cosa que su aptitud para desempeñar el trabajo en cuestión (Lee Badgett, Carpenter and Sansone, 2021). Asimismo, el derecho al trabajo implica que deben ser protegidas también las condiciones en que se desempeñan las actividades laborales (Tapiola, 2018).

Además se debe velar por la integridad física y mental de todas las personas, incluidos por supuesto los miembros del colectivo LGBTI, de manera que deben ser protegidas de todas las formas de malos tratos y abusos como el acoso laboral entre otros (López, 2017). Hay que señalar que en México el derecho al trabajo está protegido tanto ante las instituciones públicas como ante las instituciones privadas: "esta determinación ha sido confirmada por Tribunales Mexicanos, al establecerse que el principio de no discriminación rige no solo para las autoridades sino también para los particulares" (Suprema Corte de Justicia de la Nación, 2014, p. 81).
Respecto al impacto que deben tener estas normas nacionales e internacionales dentro de las organizaciones, la Oficina del Alto Comisionado para los Derechos Humanos publicó en septiembre de 2017 el reporte Tackling Discrimination against Lesbian, Gay, Bi, Trans, \& Intersex People. Standards of Conduct for Business. Se trata de una guía para que las empresas no solo eviten la discriminación y la violencia contra las personas LGBTI sino que contribuyan activamente en la garantía de todos sus derechos. Los cinco estándares que las Naciones Unidas recomiendan a las empresas, que además están pensados para orientar las políticas y operaciones de las empresas en los distintos momentos y ámbitos de sus actividades, son los siguientes: en todo momento: 1 . Respete los derechos humanos. En los lugares de trabajo: 2 . Elimine la discriminación, 3. Provea apoyo. En las actividades de trabajo: 4. Prevenga otras violaciones contra los derechos humanos. En la comunidad: 5. Influya en la esfera pública (OHCHR, 2017).

Lamentablemente, y como se ha señalado, la realidad laboral que se vive dentro de las empresas no siempre se ajusta al marco regulatorio establecido por los Estados; y a pesar del esfuerzo llevado a cabo internacionalmente sigue habiendo incertidumbre frente a la realidad laboral de ciertos colectivos (Salomon, 2014). Sin embargo la hipótesis que motiva este artículo es que las empresas de nacionalidad correspondiente a países que cuentan con este tipo de normativas deberían incluir dentro de sus políticas laborales la protección por orientación sexual e identidad de género, lo cual tendría que considerarse en sus diferentes ubicaciones en caso de ser transnacionales. Lo anterior, puede llegar a ser muy valioso si se considera que la amplitud territorial que suelen tener algunas organizaciones hace que tengan presencia en ciertos países y regiones en las que este tipo de leyes no han conseguido ser adoptadas por las normas nacionales.

\section{Metodología}

El presente artículo tiene como objetivo hacer un análisis de contenidos sobre la implementación de normas relacionadas con el derecho a la no discriminación por motivo de identidad de 
género en reportes de sostenibilidad de empresas transnacionales firmantes del Pacto Mundial con representación en México, con el fin de identificar una posible relación entre las políticas laborales y el marco regulatorio de su país de origen.

Para ello se plantea la siguiente hipótesis:

H1: la presencia de normas laborales relacionadas con el derecho a la no discriminación poridentidad degénero en empresastransnacionales se relaciona con la normativa de su país de origen.

Para los fines de este artículo se han considerado los siguientes elementos al momento de seleccionar la muestra: (i) empresas transnacionales con presencia en México; (ii) que el país deorigen de estas empresas posea leyes laborales que protejan contra la discriminación por orientación sexual e identidad de género; (iii) que sean empresas firmantes del Pacto Mundial y que se encuentren al día en sus reportes de sostenibilidad. A partir de estos criterios se han podido seleccionar 16 organizaciones de 9 nacionalidades distintas (tabla 4).

Tabla 4. Muestra de empresas

\begin{tabular}{|c|c|}
\hline País & Empresa \\
\hline Bélgica & Anheuser Busch \\
\hline Australia & $\mathrm{BHP}$ \\
\hline \multirow[t]{3}{*}{ Suecia } & Ericsson \\
\hline & Volvo \\
\hline & Scania \\
\hline \multirow[t]{3}{*}{ Reino Unido } & Unilever \\
\hline & Diageo \\
\hline & HSBC \\
\hline \multirow[t]{2}{*}{ Finlandia } & KONE \\
\hline & Nokia \\
\hline Republica Checa & IBA Group \\
\hline \multirow[t]{3}{*}{ Francia } & L'Oreal \\
\hline & Schneider Electric \\
\hline & Danone \\
\hline \multirow[t]{2}{*}{ Noruega } & Equinor \\
\hline & Norsk Hydro \\
\hline
\end{tabular}

Fuente: elaboración propia por parte de los autores.
Considerando la amplitud de información que significa la revisión de los reportes de sostenibilidad y otros documentos accesorios relacionados con políticas laborales de estas empresas, el presente artículo se ha desarrollado por medio de la adopción de una metodología de análisis de contenidos.

Según Drisko y Maschi (2016) la utilización del análisis de contenidos como herramienta de investigación ayuda a identificar la presencia de conceptos, nociones, palabras o ideas en textos o contenidos en general; esto permite cuantificar y analizar no solo su presencia sino sus significados y las relaciones que estas tienen con otras nociones, conceptos o palabras; lo que conlleva, después, a hacer inferencias sobre los mensajes que se reflejan en los mismos. A lo anterior, se le denomina análisis relacional de contenidos (Hsieh and Shannon, 2005).

La selección de esta metodología se puede justificar al considerar la necesidad de contar con una herramienta de estudio que permita analizar los diferentes documentos organizacionales, haciendo relaciones entre los patrones que se repitan en ellos. Esto permitirá hacer una reflexión que lleve a la comprobación de la hipótesis.

Para poder implementar este análisis relacional de contenidos se ha hecho una búsqueda de información a partir de la identificación de palabras clave relacionadas con el tema tanto en español como en inglés: orientación sexual; identidad de género; expresión de género; afirmación o confirmación de género; transgénero; transexual; trans; LGBTI/ GLBT; identidad no binaria e intersexuales.

Esta clasificación de palabras se construyó a partir de la propuesta que hace el WORLD Policy Analysis Center, así como de los mapas de legislación sobre orientación sexual de la Asociación Internacional de Lesbianas, Gays, Bisexuales, Trans e Intersexuales -ILGA-.

\section{Resultados y discusión}

Una vez que se llevó a cabo el análisis de la información de cada una de las empresas de la muestra, a partir de la categoría planteada en 
la metodología, fue posible dividirlas según su nivel de compromiso institucional (tabla 5); allí se consideraron las empresas que contaban con normas generales contra la discriminación, las empresas con normas contra la discriminación por motivos de orientación sexual y las empresas con normas contra la discriminación por motivo de orientación sexual e identidad de género.

Tabla 5. Resultados del análisis de contenido

\begin{tabular}{|c|c|c|c|}
\hline Empresa & $\begin{array}{c}\text { Normas generales } \\
\text { contra la discriminación }\end{array}$ & $\begin{array}{c}\text { Normas contra la } \\
\text { discriminación por motivo } \\
\text { de orientación sexual }\end{array}$ & $\begin{array}{c}\text { Normas contra la discriminación } \\
\text { por motivo de orientación sexual e } \\
\text { identidad de género }\end{array}$ \\
\hline Anheuse Busch InBear & & & $x$ \\
\hline BHP & & & $x$ \\
\hline Ericsson & & & $x$ \\
\hline Volvo & & & $x$ \\
\hline Scania & & $x$ & \\
\hline Unilever & & & $x$ \\
\hline Diageo & & $x$ & \\
\hline $\mathrm{HSBC}$ & & & $x$ \\
\hline KONE & $x$ & & \\
\hline Nokia & & & $x$ \\
\hline IBA Group & $x$ & & \\
\hline L'Oreal & & & $x$ \\
\hline Schneider Electric & & & $x$ \\
\hline Danone & & $x$ & \\
\hline Equinor & & $x$ & \\
\hline Norsk Hydro & & $x$ & \\
\hline
\end{tabular}

Fuente: elaboración propia por parte de los autores.

Con base en la metodología planteada se pudo hacer una revisión de los diferentes documentos oficiales que las empresas ofrecen de manera pública sobre las políticas laborales y organizacionales que impactan en los derechos y beneficios de sus colaboradores. Entre estos documentos se revisaron los reportes de sostenibilidad que las empresas presentan como parte de su compromiso con el Pacto Mundial y que están disponibles en la página oficial de Internet de esa organización de las Naciones Unidas, así como otros documentos accesorios relacionados con políticas laborales.
Como se pudo apreciar en la tabla 5, aunque todas las empresas pertenecen a países que cuentan con legislaciones que protegen contra la discriminación por identidad de género, no todas lo reflejan en sus lineamientos laborales; esto podría ser algo explícito en las oficinas y fabricas que se encuentran en su país de origen, pero no necesariamente en sus ubicaciones en otros países y regiones internacionales.

Durante el proceso de análisis de contenidos se logró identificar que la manera en que las diversas empresas expresan su posición ante la discriminación no siempre se correlacionan con 
la normativa de sus países de origen; ya que, aunque todas incluyen cláusulas contra cualquier tipo de conducta discriminatoria, no todas hacen una mención expresa y literal sobre la discriminación por identidad de género.

Casos como KONE e IBA Group mencionan una prohibición general contra la discriminación de cualquier índole y un apego al respeto a los derechos humanos; esto, aunque se podría decir que cubre la identidad de género, no es para nada claro; lo anterior, deja espacio a interpretaciones en sus ubicaciones fuera de sus países de origen.

Por su parte Scania, Diageo, Danone, Equinor y Norsk Hydro incluyen un señalamiento expreso contra la discriminación por orientación sexual, pero no lo hacen por identidad de género; esto, según lo señalado por el WORLD Policy Analysis Center, significa una diferencia. En cuanto a las demás empresas, todas incluyen un señalamiento expreso hacia la no discriminación por identidad de género; considerando las diferentes variaciones que se señalaron en la metodología del análisis realizado.

Es importante mencionar que hay dos situaciones que no resultan ser claras para la metodología propuestay que podrían ser limitantes del presente texto. La primera de ellas tiene relación con la mención de políticas que protegen contra la discriminación a personas LGBTI tal como es el caso de HSBC, lo cual en un sentido amplio cubre la identidad de género; aunque estrictamente hablando es un señalamiento general que no específica si se refiere a ella o solo a la orientación sexual. El otro caso es el relacionado con aquellas empresas a las que se pudo tener acceso a reportes de sostenibilidad y políticas laborales nacionales como es el caso de Scania México y de Schneider Electric México. En el caso de Scania es interesante remarcar que el reporte presentado por su filial mexicana es detallado y con apego a la no discriminación por orientación sexual, lo cual no se menciona en el reporte internacional. En el caso de Schneider, mientras el reporte internacional señala literalmente la protección de personas trans e intersexuales, en el reporte mexicano solo se hace mención a la sensibilidad con respecto a la comunidad LGBTI sin dar mayores detalles.

Todo ello permite tener información suficiente para considerar que la hipótesis planteada es negativa; pues, a pesar de que las empresas consideradas en la muestra provienen de países en los que existe una legislación laboral en contra de la discriminación por identidad de género, esto no se refleja en las políticas laborales en todos los casos y por lo tanto no garantiza que sea algo que se adopte en sus filiales en otros países; además de que no se ajustan tampoco a las exigencias de la legislación mexicana.

Lamentablemente, aunque se puede apreciar un claro interés de las organizaciones por ser cada vez más responsables y atender a estas necesidades sociales, aún no es posible garantizar que estas medidas sean adoptadas de manera oficial dentro de sus políticas y normativas; lo anterior, da un resultado negativo en la hipótesis propuesta.

\section{Conclusiones}

No cabe duda de que el esfuerzo internacional por promover el derecho al trabajo está cada día más presente en la agenda de los países; aunque las normativas nacionales sean más conscientes de la importancia de la inclusión y la no discriminación por motivo de la orientación sexual, la identidad de género, la expresión de género y las características sexuales, esto no garantiza su adopción e implementación dentro de las organizaciones de manera íntegra. Por si esto no fuera ya un reto por sí mismo, siguen existiendo claras lagunas dentro de la legislación que protege a los individuos laboralmente dealgunos países y que dejan a grupos completos (como, por ejemplo, la comunidad trans) en un estado de indefensión e incertidumbre.

En aras de dar luz a esta problemática, la presente investigación presenta el nivel de impacto que pueden tener las normas laborales nacionales en las empresas multinacionales que operan fuera de las barreras territoriales de su país de origen. Como caso concreto se analizaron las políticas empresariales relacionadas con el respeto a la diversidad y a la prohibición de discriminación por motivo de identidad de género. Como conclusión se plantea que no existe una relación entre las normas laborales de las Naciones de origen con las políticas laborales propias de las organizaciones, no pudiendo confirmarse el mismo compromiso 
hacia el respeto de los derechos laborales de los miembros del colectivo LGBTI.

A pesar de que se ha intentado hacer una reflexión a partir de empresas firmantes del Pacto Mundial por el compromiso con los derechos humanos que forman parte de este convenio, este estudio resulta ser limitado por la muestra tan pequeña que se ha considerado. Se reconoce que esta investigación es valiosa dado que arroja luces con respecto a esta temática, sin embargo es necesario ampliar el estudio con un mayor número de empresas multinacionales de la región o incluso considerar la situación que plantean sus políticas laborales en otros países.

Por el objetivo del presente estudio la investigación se ha enfocado en las políticas laborales relacionadas con la protección de los trabajadores por motivos de identidad de género, sin embargo es relevante ampliar este análisis a otro tipo de normas que protejan a otros grupos de los considerados vulnerables.

\section{Referencias}

ADIL. (2018). Encuesta sobre diversidad y talento LGBT en México. Ciudad de México, México: ADIL.

Alarcon Andia, D.C., Castillo Montero, C.M.y Gastelú Ledezma,I.M.(2018). Estado actual de la gestión de la diversidad LGBT en las empresas de Lima Metropolitana. Casos de estudio: IBM, Atento y Scotiabank (tesis de pregrado). Pontificia Universidad Catolica de Perú, Lima.

Badgett, M.V.L. y Sell, R. (2018). Conjunto de indicadores propuestos para el índice de inclusión LGBTI. Nueva York, Estados Unidos: PNUD.

Ball, C.A. (2016). After marriage equality: The future of LGBT rights. Nueva York, USA: New York University Press.

Brown, E. and Scott, I. (2019). Belief v. Belief: Resolving LGBTQ Righes Conglicts in the
Religious Workplace. American Business Law Journal, 56(1), 55-113.

CEDAW. (2010). Recomendación general $N^{\circ}$ 28 relativa al artículo 2 de la Convención sobre la eliminación de todas las formas de discriminación contra la mujer. Recuperado de https://www.right-to-education.org/es/ resource/cedaw-recomendaci-n-general-28.

CIDH. (2012). Orientación sexual, identidad de géneroy expresión de género: algunos términos yestándares relevantes. Recuperado de http:// www.oas.org/es/sla/ddi/docs/CP-CAJPINF_166-12_esp.pdf.

Corrales, J. (2017). Understanding the uneven spread of LGBT rights in Latin America and the Caribbean. Journal of Research in Gender Studies, 7(1), 52-82.

Dario, L.M. et al. (2019). Assessing LGBT people's perceptions of police legitimacy. Journal of Homosexuality, 67(7), 1-31.

Díaz Camarena, A. (2017). Guía para la no discriminación en la prestación de servicios a las personas LGBTTTI. Guadalajara, México: Instituto Jalisciense de las Mujeres.

Drisko, J. And Maschi, T. (2016). Content analysis. Nueva York, USA: Oxford University Press.

Flores, N. (2016). Conoce las marcas multinacionales que apoyan los derechos LGBT. Somos Gay. Recuperado de https://www.somosgay. org/noticias/detalles/conoce-las-marcasmultinacionales-que-apoyan-los-derechoslgbt.html.

Hsieh, H. and Shannon, S.E. (2005). Three approaches to qualitative content analysis. Qualitative Health Research, 15(9), 1277-1288.

Jiménez Bolaños, J.D. (2014). Temáticas en construcción: el desarrollo de los estudios LGBT en Costa Rica. Cuadernos Inter.c.a.mbio sobre Centroamérica y el Caribe, 11(2), 91-116.

Jowett, A. (2017). Representing the history of LGBT rights: political rhetoric surrounding the 50th 
anniversary of the Sexual Offences Act 1967. Psychology \& Sexuality, 8(4), 306-317.

Keck, T.M. (2009). Beyond backlash: Assessing the impact of judicial decisions on LGBT rights. Law \& Society Review, 43(1), 151-186.

Lamond, I.R. (2018). The challenge of articulating human rights at an LGBT mega event: a personal reflection on Sao Paulo Pride 2017. Leisure Studies, 37(1), 36-48.

Lee Badgett, M.V., Carpenter, C.S. and Sansone, D. (2021). LGBTQ Economics.Journal of Economic Perspectives, 35(2), 141-170.

López, J.A. (2017). Los derechos LGBT en México: acción colectiva a nivel subnacional. European Review of Latin American and Caribbean Studies, 104, 69-88.

Margarit, D. (2019). LGBTQ rights, conservative backlash and the constitutional definition of marriage in Romania. Gender, Place \& Culture, 26(11), 1570-1587.

Matthews, P. (2020). Debate: LGBTQ rights in public services-a battle won? Public Money \& Management, 40(6), 423-425.

OACDH. (2013). Orientación sexual e identidad de género en el derecho internacional de los derechos humanos. Santiago de Chile, Chile: $\mathrm{OACDH}$.

OHCHR. (2017). Tackling Discrimination against Lesbian, Gay, Bi, Trans \& Intersex People. Standards of Conduct for Business. Geneva, Switzerland: OHCHR.

OHCHR. (2019). Born Free and Equal. Sexual Orientation, Gender Identity and Sex Characteristics in International Human Rights Law. Geneva, Switzerland: OHCHR.

Principios de Yogyakarta. (2007). Principios sobre la aplicación de la legislación internacional de derechos humanos en relación con la orientación sexual y la identidad de género. Recuperado de http://yogyakartaprinciples.org/wp-content/ uploads/2016/08/principles_sp.pdf.
Salomon, A. (2014). Identity or Behavior: A Moral and Medical Basis for LGBTQ Rights. The Hastings Center Report, 44(5), S4-S5.

Szyndlar, M. and Wasikiewicz-Firlej, E. (2019). The LGBT Community as a stakeholder in communicating corporate social responsability. Scripta Neophilologica Posnaniensia, 19, 191-121.

Suprema Corte de Justicia de la Nación. (2014). Protocolo de actuación para quienes imparten justicia en casos que involucren la orientación sexual o la identidad de género. Ciudad de México, México: Suprema Corte de Justicia de la Nación.

Tapiola, K. (2018). La declaración relativa a los principios y derechos fundamentales en el trabajo de 1998. Una poderosa herramienta de la OIT. Ginebra, Suiza: OIT.

Vijlbrief, A., Saharso, S. And Ghorashi, H. (2020). Transcending the gender binary: Gender nonbinary young adults in Amsterdam. Journal of LGBT Youth, 17(1), 89-106.

Wilson, B.M. and Gianella-Malca, C. (2019). Overcoming the limits of legal opportunity structures: LGBT rights divergent paths in Costa Rica and Colombia. Latin American Politics and Society, 61(2), 138-163.

WORLD Policy Analysis Center. (2020). Are LGBT workers protected from discrimination in promotions and/or demotions? Recuperado de https://ph.ucla.edu/are-lgbt-workersprotected-discrimination-promotions-andordemotions. 